\title{
Comparison of caspase-3 activation in tumor cells upon treatment of chemotherapeutic drugs using capillary electrophoresis
}

\author{
Shuang Sha ${ }^{1,2}$, Honglin $\mathrm{Jin}^{1,2}$, Xiao $\mathrm{Li}^{1,2}$, Jie Yang ${ }^{1,2}$, Ruiting $\mathrm{Ai}^{3}$, Jinling $\mathrm{Lu}^{1,2} \bowtie$ \\ ${ }^{1}$ Britton Chance Center for Biomedical Photonics-Wuhan National Laboratory for Optoelectronics, Huazhong University of \\ Science and Technology, Wuhan 430074, China \\ 2 Key Laboratory of Biomedical Photonics of Ministry of Education, Huazhong University of Science and Technology, \\ Wuhan 430074, China \\ ${ }^{3}$ China National Center for Biotechnology Development, Beijing 100036, China \\ $\triangle$ Correspondence: lujinling@mail.hust.edu.cn \\ Received November 17, 2011 Accepted January 5, 2012
}

\section{ABSTRACT}

Caspases play important roles in cell apoptosis. Measurement of the dynamics of caspase activation in tumor cells not only facilitates understanding of the molecular mechanisms of apoptosis but also contributes to the development, screening, and evaluation of anticancer drugs that target apoptotic pathways. The fluorescence resonance energy transfer (FRET) technique provides a valuable approach for defining the dynamics of apoptosis with high spatio-temporal resolution. However, FRET generally functions in the single-cell level and becomes ineffective when applied in the high throughput detection of caspase activation. In the current study, a FRET sensor was combined with capillary electrophoresis (CE) to achieve a high throughput method for cellular caspase detection. The FRET-based CE system is composed of a homemade CE system and a laser source for detecting the dynamics of caspase-3 in various cells expressing sensors of caspase- 3 that have been treated with anticancer drugs, such as cell cycle-independent drug cisplatin and specific cell cycle drugs camptothecin and etoposide, as well as their combination with tumor necrosis factor (TNF). A positive correlation between the caspase- 3 activation velocity and drug concentration was observed when the cells were treated with cisplatin, but cells induced by camptothecin and etoposide did not show any apparent correlation with their concentrations. Moreover, different types of cells presented distinct sensitivities under the same drug treatment, and the combination treatment of TNF and anticancer drugs significantly accelerated the caspase- 3 activation process. Its high throughput capability and detection sensitivity make the FRET-based CE system a useful tool for investigating the mechanisms of anticancer drugs and anticancer drug screening.

KEYWORDS apoptosis, caspase-3, fluorescence resonance energy transfer (FRET), capillary electrophoresis (CE)

\section{INTRODUCTION}

Apoptosis plays important roles in maintaining the development of multicellular organisms. Apoptosis defects give rise to both tumorigenesis and drug resistance, leading to failures in the clinical chemotherapy of cancers (Johnstone et al., 2002). Evidence shows that the screens of apoptosis are superior to the proteins in cells for anticancer drug screening (Joseph et al., 2011). Caspases, which decide the morphological and biochemical reactions of organisms, mediate apoptosis. Cysteine caspases are inactive zymogens in cells that are activated by an apoptotic stimulus. As an effector of apoptosis, caspase- 3 activation has been accepted as a reliable readout for apoptosis, and proteinase activity has been widely exploited for drug screening (Luo et al., 2003).

Many approaches can be used for the assessment of caspase activity, including traditional SDS-PAGE, Western blot, and high-performance liquid chromatography. However, these methods do not give information on the dynamics of 
caspase activation. Over the last decade, the development of novel methods for assaying the activation of various types of caspases in single living cells has received significant attention (Mahajan et al., 1999; Jones et al., 2000; Tyas et al., 2000; Neefjes and Dantuma, 2004; Zhang et al., 2008a). The common idea of previous studies is the construction of a fluorescence resonance energy transfer (FRET) probe based on fluorescent proteins (FPs). The FP-FRET probe is composed of two separate spectra of fluorescent proteins, such as CFP and YFP, which are sandwiched with the recognized sequence of the specific caspase. Based on this idea, two caspase FP-FRET probes, named CD2 and CD3, consisting of CFP, DsRed (a red fluorescent protein), and a linker with recognition sites of caspase-2 and caspase-3, respectively, were developed (Lin et al., 2006; Zhang et al., 2008b). Such probes are highly useful for studying the fundamental mechanisms of apoptosis and can serve as powerful tools for screening new drug candidates targeted to apoptotic pathways. FP-FRET probes coupled with time-lapse confocal microscopic imaging have given valuable insights into cellular events within single cells with high spatio-temporal resolution (Yang et al., 2007; Zhang et al., 2008b). However, single cell-based studies using FP-FRET probes and microscopic imaging techniques cannot describe the high throughput dynamics of caspase activation in tumor cells upon drug treatment. FRET-based live cell detection systems have either failed to give quantitative information or are not adaptable for multi-parameter analyses.

Zhou et al. recently developed a novel strategy to characterize the dynamics of caspase-2 activation using the FPFRET probe CD2 combined with capillary electrophoresis (CE) technology (Zhou et al., 2006a). CE, which has advantages of high speed, low sample consumption, and automation, has been developed as a mature tool for sample enrichment, separation, and detection (Locke and Figeys, 2000). Moreover, the use of CE to monitor changes in protease activity has been extensively studied (Berezovski et al., 2002; Zarrine-Afsar and Krylov, 2003), with results indicating that CE is an effective method for detecting changes in protease activity. The dynamics of caspase- 3 activation in Hela cells upon cisplatin treatment have been previously investigated by combining the FRET probe with CE (Lin et al., 2006). However, data on the activation characteristics of caspase- 3 in different cells and the exposure of caspase- 3 on various drug treatment schemes remain insufficient. Besides the Hela cell, HepG2 and ACC-M cells are also human cancer cells commonly used for drug screening (Chen and Tsourka, 2009; Zhu et al., 2009). In the current study, cisplatin, camptothecin, and etoposide were selected for cell treatment because of their different characteristics. Cisplatin is a non-specific cell cycle drug, whereas camptothecin and etoposide are specific cell cycle drugs (Chen and Liu, 1994; Zuryn et al., 2007). The description of the dynamics of apoptosis in different tumor cells after treatment with varied drug treatment schemes will be helpful in assessing the effects of different therapeutic schemes and understanding drug resistance in cancer chemotherapy.

The current study aims to use the established FRET-CE system to investigate the dynamics of caspase- 3 during apoptosis in cancer cells. This study provides valuable insights into the molecular mechanisms of caspase- 3 during cell apoptosis induced by anticancer drug treatment.

\section{RESULTS}

\section{Quantification of caspase-3 activation in Hela cells upon drug treatments}

Different concentrations of drugs were used to induce the apoptosis of Hela-CD3 cells and optimize the concentrations of drugs for activating caspase- 3 in cells. Figure $1 \mathrm{~A}$ shows the electropherogram results of Hela-CD3 cells $13 \mathrm{~h}$ after treatment with different concentrations of cisplatin. Caspase- 3 was activated during cell apoptosis and digested the substrate sequences of CD3 and DsRed fluorescent proteins, which were cut from CD3 during cisplatin treatment. DsRed was eluted out first during electrophoresis (peak 1 in Fig. 1A) followed by intact CD3 (peak 2 in Fig. 1A) because the electron mass of DsRed is smaller than that of CD3. The intensity of DsRed was divided into the intensities of the detached DsRed and CD3 (peaks 1 and 2 in Fig. 1A) for the evaluation of caspase- 3 activation, and the ratios of the DsRed intensities from the detached DsRed and intact CD3 were calculated; these ratios represented the states of caspase- 3 activation (Fig. 1B). The ratio of intensities increased with increasing cisplatin concentration, suggesting that CD3 is digested gradually and the caspase- 3 activation becomes more and more efficient. A strong positive linear correlation $\left(R^{2}=\right.$ $0.995)$ was found between caspase- 3 activation and the experimental cisplatin concentrations $(0-67 \mu \mathrm{mol} / \mathrm{L})$, as shown in Fig. 1B.

The same method was then used to evaluate the effects of camptothecin and etoposide on caspase- 3 activation in Hela-CD3 cells. A series of concentrations of camptochecin and etoposide were used to induce the apoptosis of Hela-CD3 cells over 24 and $48 \mathrm{~h}$. Caspase-3 activation was detected using the CE system and quantified using the ratio of DsRed intensities from isolated DsRed and intact CD3 molecules (Fig. 2).

Unlike the linear relationship presented in the cisplatin treatment group (Fig. 1), caspase-3 activity did not increase significantly with increasing concentrations of camptothecin and etoposide after $24 \mathrm{~h}$ of incubation, as shown by the dotted lines in Fig. 2. While caspase-3 activation was significantly higher after the $48 \mathrm{~h}$ incubation period, activity did not significantly increase with increasing concentrations of the drugs either, as shown by the solid lines in Fig. 2. These results are likely due to the effects of cisplatin, which is gen- 
A

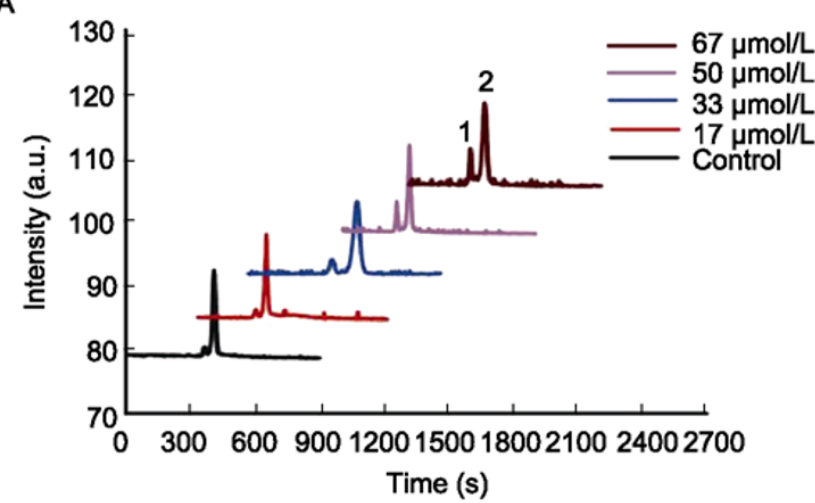

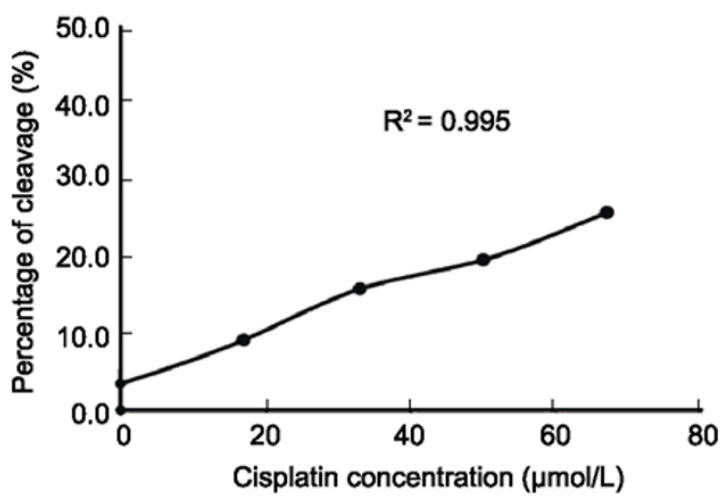

Figure 1. Detection of caspase-3 activation in Hela cells incubated with cisplatin using capillary electrophoresis. (A) Electropherograms of lysates of Hela-CD3 cells incubated with different concentrations of cisplatin for $13 \mathrm{~h}$. Peak identities: (1) DsRed and (2) CD3. (B) The ratios of the intensities of the DsRed and CD3 peaks in (A) were calculated; these ratios represent the states of caspase-3 activation in Hela cells under treatment with different concentrations of cisplatin.

A

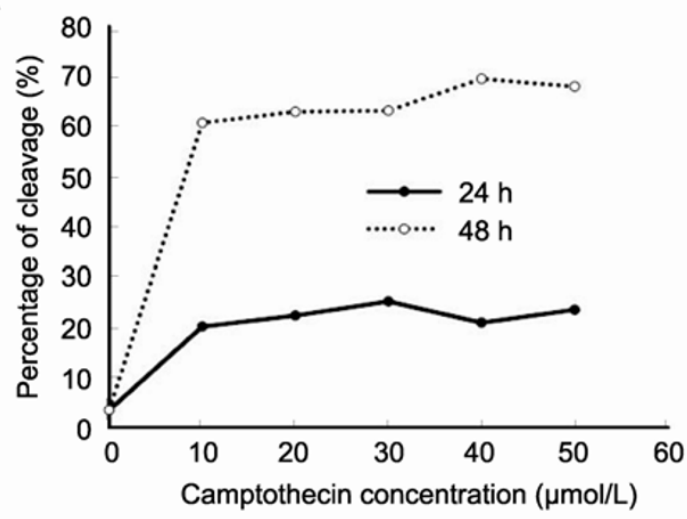

B

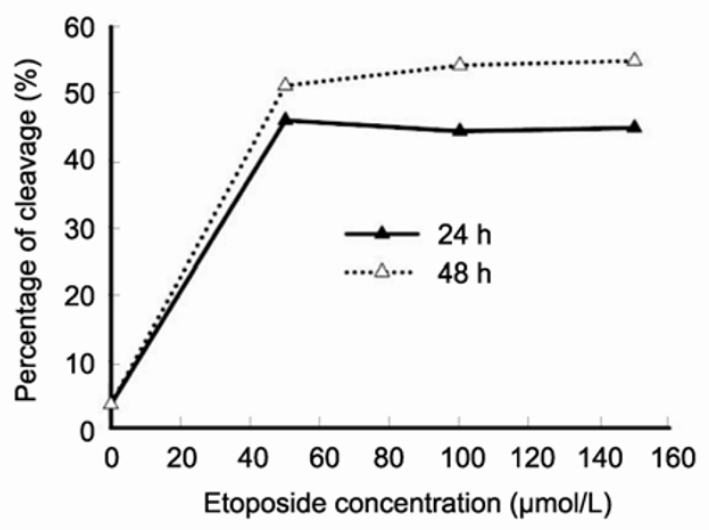

Figure 2. Caspase-3 activation of Hela cells incubated with different concentrations of (A) camptothecin and (B) etoposide for 24 and $48 \mathrm{~h}$.

erally considered as a cell cycle non-specific anticancer drug and is a major antineoplastic drug used for the treatment of solid tumors in any phase of cell proliferation (Yao et al., 2007). In contrast, camptothecin and etoposide are DNA topoisomerase I and II inhibitors, respectively (Bergeron et al., 2004; Abbott et al., 2009). DNA topoisomerases are essential nuclear enzymes with multiple functions, including chromosomal recombination and DNA repair (de Jonge et al., 2004). Camptothecin and etoposide are specific cell-cycle phase agents (Hsu et al., 2009). Camptothecin shows cell-cycle S phase specificity, whereas etoposide induces cell-cycle arrest at the $G_{2}$ phase (Chen and Liu, 1994; Zuryn et al., 2007). Cellular caspase-3 activation in other phases may take a longer time to occur because of the transition time from other phases into the $\mathrm{S}$ or $\mathrm{G}_{2}$ phase. Therefore, caspase-3 activation processes induced by camptothecin and etoposide are much slower than that induced by cisplatin. Further increases in concentration do not hasten the apoptosis process of cells when the drug concentration reaches the minimum value that can exert the most effective equilibration for the $S$ and $G_{2}$ phase cells. Caspase-3 activation was significantly higher after $48 \mathrm{~h}$ of incubation than that of $24 \mathrm{~h}$ because more cells are able to differentiate into the $S$ or $G_{2}$ phase with longer incubation periods.

The results obtained thus far suggest that the velocity of cisplatin-induced caspase- 3 activation has a positive correlation with its concentration under the experimental concentration range and that activation induced by camptothecin or etoposide shows no apparent correlation with concentration. Therefore, optimal cisplatin, camptothecin, and etoposide concentrations of 67,30 , and $50 \mu \mathrm{mol} / \mathrm{L}$, respectively, were selected for further experiments to achieve potent caspase- 3 
activation while maintaining a low drug dose.

\section{Different cells show various sensitivities to chemotherapeutic drugs in caspase- 3 activation}

Three stable cell lines (i.e. Hela-CD3, HepG2-CD3, and ACC-M-CD3) were individually incubated with $67 \mu \mathrm{mol} / \mathrm{L}$ of cisplatin, $30 \mu \mathrm{mol} / \mathrm{L}$ of camptothecin, or $50 \mu \mathrm{mol} / \mathrm{L}$ of etoposide to assess the effects of anticancer drugs on different types of cells. The cell samples were collected and were subjected to electrophoresis after incubation with drugs for a designated period of time. Figure $3 \mathrm{~A}$ shows the time courses of caspase- 3 activation in the three cell lines upon treatment with cisplatin. At the beginning of caspase- 3 activation, the three types of cells showed no obvious differences. As activation progressed, the ACC-M-CD3 cell line was a little faster. After incubation, the HepG2-CD3 cell line was faster than the ACC-M and the Hela-CD3 cell line was the slowest. These results demonstrate that the HepG2 cell line is more sensitive to $67 \mu \mathrm{mol} / \mathrm{L}$ cisplatin than the other two cell lines during cell apoptosis. As expected, the cell lines displayed distinct responses after treatment with camptothecin and etoposide compared with cisplatin (Fig. 3B and $3 \mathrm{C}$ ). When exposed to $30 \mu \mathrm{mol} / \mathrm{L}$ of camptothecin, HepG2 cells showed the most rapid dynamic change in caspase- 3 activity, with complete cleavage of CD3 after $30 \mathrm{~h}$ of incubation, whereas Hela and
ACC-M cells achieved complete cleavage of CD3 after 54 and $60 \mathrm{~h}$ of incubation, respectively. A similar trend was observed for the etoposide treatment group (Fig. 3C), in which HepG2, ACC-M, and Hela cells achieved complete cleavage of the CD3 sensor after 42, 60, and $66 \mathrm{~h}$ of incubation, respectively. Overall, the results show that the CE system can trace the dynamics of caspase-3 activity after drug treatment and suggest the distinct sensitivities of different cell lines to the drugs.

\section{Dynamics of caspase-3 activation of three cell lines after treatment with TNF and drugs}

Cytokine tumor necrosis factor-alpha (TNF- $\alpha$ ) is highly expressed in tumors and promotes the growth of new capillaries toward the tumors (Burton and Libutti, 2009). However, TNFcan be used to destroy tumor vasculature when it is accumulated at high doses (Burton and Libutti, 2009). TNF- $\alpha$ can initiate the process of cellular apoptosis, and these pathways can deactivate the growth of tumor cells (Szlosarek and Balkwill, 2003). In addition, the combination of TNF- $\alpha$ with anti-tumor drugs may have strong synergistic anti-tumor efficacy. Hela-CD3 cells were subjected to treatments with TNF $\left(1 \times 10^{4} \mathrm{IU} / \mathrm{mL}\right)$ alone, drugs alone, and TNF in combination with drugs and then monitored by CE to observe the effect of TNF on the destruction of tumor cells (Fig. 4). Only part of the
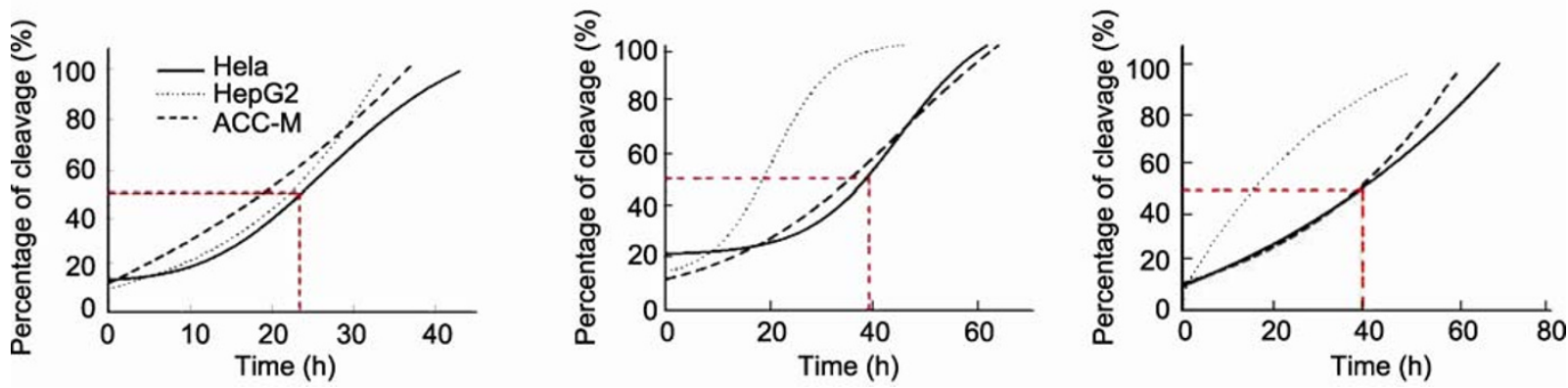

Figure 3. Time courses of caspase-3 activation in Hela, HepG2, and ACC-M cell lines incubated with (A) cisplatin, (B) camptothecin, and (C) etoposide.

A

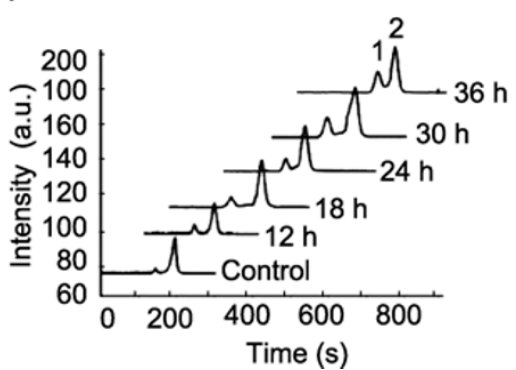

B

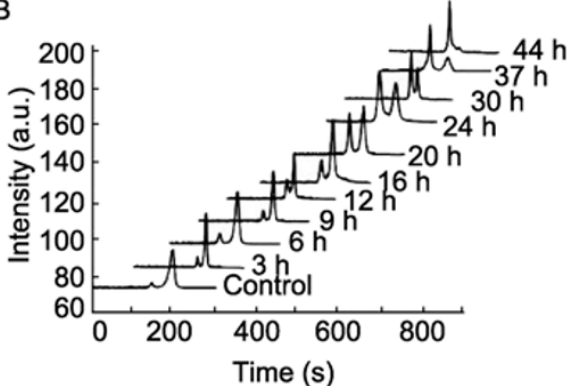

C

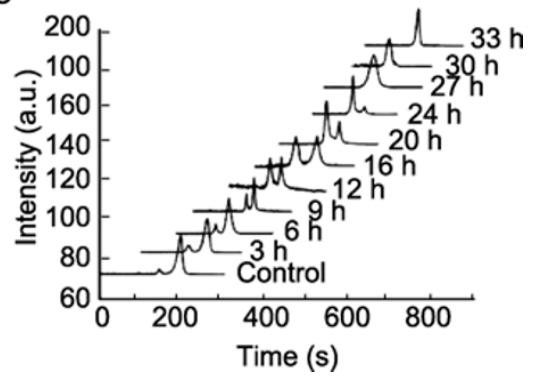

Figure 4. Electropherograms of lysates of Hela-CD3 cells incubated with tumor necrosis factor (TNF) and cisplatin in a time course. (A) Hela-CD3 cells were incubated with $1 \times 10^{4} \mathrm{IU} / \mathrm{mL}$ of TNF alone for 12 to $36 \mathrm{~h}$. (B) Hela-CD3 cells were incubated with $20 \mu \mathrm{g} / \mathrm{mL}$ of cisplatin alone for 3 to $44 \mathrm{~h}$. (C) Hela-CD3 cells were incubated with $1 \times 10^{4} \mathrm{IU} / \mathrm{mL}$ of TNF $+67 \mu \mathrm{mol} / \mathrm{L}$ of cisplatin for 3 to $33 \mathrm{~h}$. 
DsRed proteins from the CD3 probes were cleaved when the Hela-CD3 cells were treated with TNF for $36 \mathrm{~h}$ (Fig. 4A), indicating that $1 \times 10^{4} \mathrm{IU} / \mathrm{mL}$ TNF is not cytotoxic to the cells under the conditions of the current experiment. Peaks 1 and 2 represent the cleaved product of DsRed and the uncleaved CD3 probe, respectively. Complete cleavage of the CD3 probes occurred after $44 \mathrm{~h}$ of incubation when the Hela cells were treated with cisplatin (Fig. 4B). Complete cleavage of the CD3 probes was observed after $27 \mathrm{~h}$ of incubation when the cells were treated with TNF + cisplatin, $17 \mathrm{~h}$ ahead of treatment with cisplatin alone (Fig. 4C). This enhanced cleavage rate indicates the synergistic effects of cisplatin and
TNF in a single treatment.

The dynamics of caspase- 3 activation in the three cell lines under various treatment conditions was then analyzed (Fig. 5). Figures 5A-5C show the dynamics of caspase-3 activation in the three cell lines under treatment with cisplatin alone, TNF alone, and TNF + cisplatin. As can be seen in the figures, the caspase- 3 activation rate in the TNF + cisplatin-treated group was much higher than those in the TNF-alone or cisplatin-alone treatment groups in all cell lines. Similar results were obtained in the TNF + camptothecin (Fig. 5D-5F) and TNF+ etoposide (Fig. 5G-5I) groups. Such results demonstrate the caspase- 3 activation rate is much
A

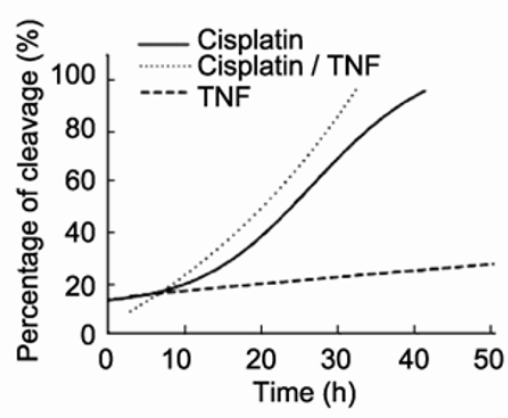

D

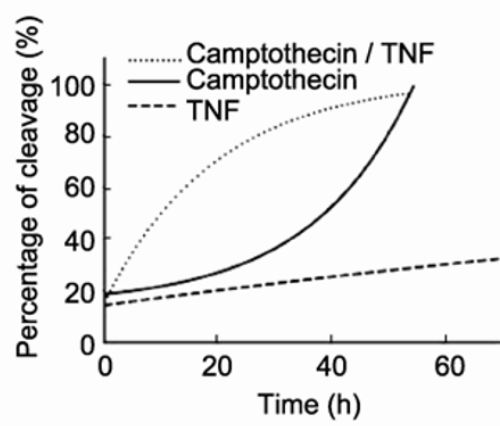

G

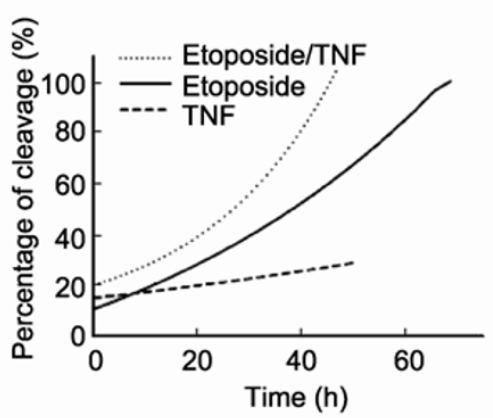

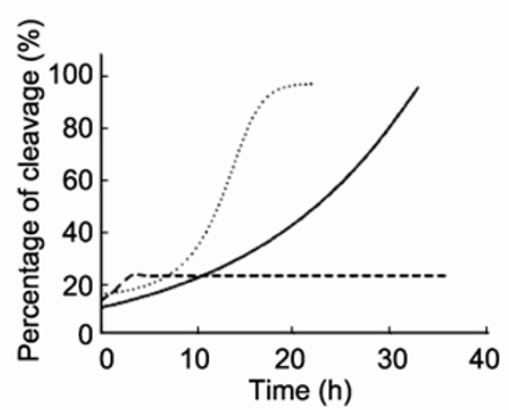

E

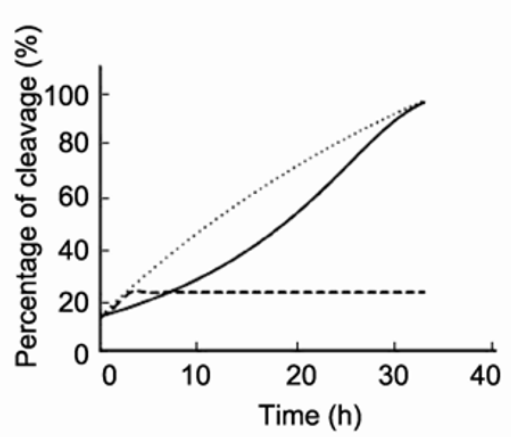

$\mathrm{H}$

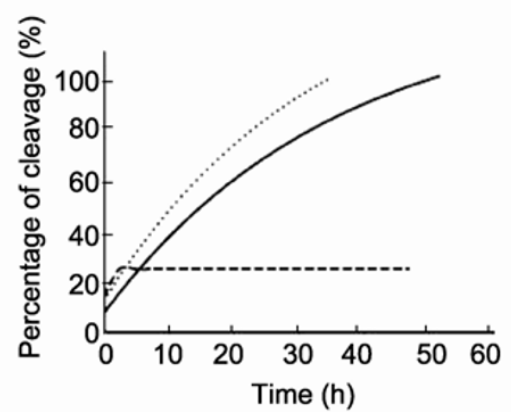

C

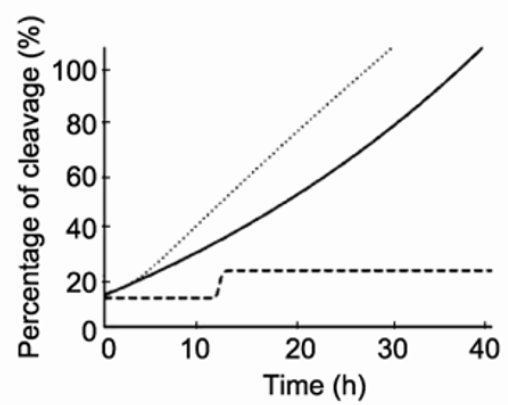

$\mathrm{F}$

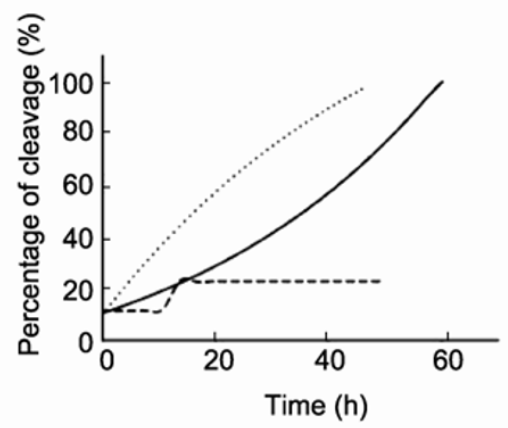

I

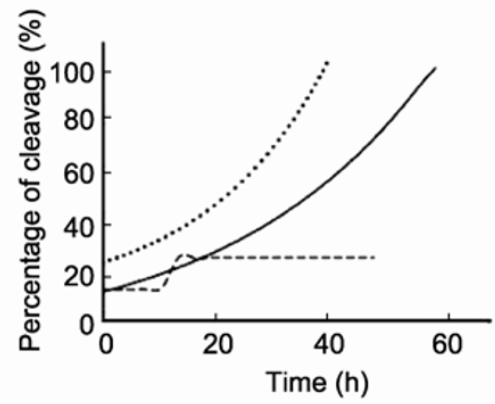

Figure 5. Time courses of caspase-3 activation in different cells incubated with TNF and different drugs. Time courses of caspase- 3 activation in (A) Hela cells, (B) HepG2 cells, and (C) ACC-M cells incubated with $1 \times 10^{4} \mathrm{IU} / \mathrm{mL}$ of TNF alone, $67 \mu \mathrm{mol} / \mathrm{L}$ of cisplatin alone, and TNF + cisplatin. Time courses of caspase- 3 activation in (D) Hela cells, (E) HepG2 cells, and (F) ACC-M cells incubated with $1 \times 10^{4} \mathrm{IU} / \mathrm{mL}$ of TNF alone, $30 \mu \mathrm{mol} / \mathrm{L}$ of camptothecin alone, and TNF + camptothecin. Time courses of caspase-3 activation in (G) Hela cells, (H) HepG2 cells, and (I) ACC-M cells incubated with $1 \times 10^{4} \mathrm{IU} / \mathrm{mL}$ of TNF alone, $50 \mu \mathrm{mol} / \mathrm{L}$ of etoposide alone, and TNF + etoposide. 
higher in TNF + drug combination groups than in groups treated with either TNF alone or drugs alone. Compared with individual drug treatments, TNF + cisplatin and TNF + etoposide enhanced apoptosis in the later stages of the process (Fig. 5A-5C and Fig. 5G-5I, respectively). In contrast, TNF + camptothecin significantly promoted caspase-3 activation at the beginning of incubation (Fig. 5D-5F).

\section{DISCUSSION}

Many anticancer drugs have been developed to suppress cancer by activating caspases because caspases play key roles in apoptosis. Measurement of the dynamics of caspase activation in tumor cells facilitates understanding of the molecular mechanisms of apoptosis, as well as the screening and evaluation of anticancer drugs targeted to apoptotic pathways. In the current study, the dynamic characteristics of caspase-3 activity in different cells over a range of treatment strategies were conveniently compared for the first time using a homemade FRET-CE system.

Cisplatin, camptothecin, and etoposide are chemotherapeutic agents commonly used for treating various tumors, including lung, breast, ovarian, colorectal, and testicular cancers (Reinhold et al., 2003). Many tumor cells have been reported to undergo apoptotic cell death when treated with the drugs described above (Tsuruo et al., 2003). However, the mechanisms of these drugs differ from one another. Camptothecin and etoposide are cell cycle-specific drugs that affect the $S$ phase of cell proliferation, whereas cisplatin is a cell cycle non-specific drug whose effectiveness does not depend on any proliferation phase. Therefore, the use of camptothecin and etoposide for the induction of cellular caspase- 3 was not as effective as the treatment with cisplatin (Fig. 3). ACC-M cells are more sensitive to cisplatin than other cells (Fig. 3A), whereas HepG2 cells appear to be more sensitive to camptothecin and etoposide (Fig. $3 B$ and $3 C$, respectively).

Combination therapy using TNF and anticancer drugs was recently promoted in many clinics as an effective method for the treatment of various cancers. The advantages of using combination therapy appear to lie in its combined targeting ability to different signal transduction pathways and enhanced approaches for combating drug resistance (Postiglione et al., 2011). Richard (Hara et al., 2005) reported that combination of low concentrations of TNF $\left(1 \times 10^{2} \mathrm{IU} / \mathrm{mL}\right)$ and cisplatin has no obvious effects on caspase- 3 activation. The results of the current study demonstrate that high concentrations of TNF $\left(1 \times 10^{4} \mathrm{IU} / \mathrm{mL}\right)$ and cisplatin can promote caspase- 3 activation in Hela-CD3 cells (Fig. 4) and that this enhanced effect was not affected by the individual role of each drug (Fig. 4A). However, the dynamics of enhancement is drug dependent. TNF enhanced apoptosis during the late stages of the caspase-3 activation processes of cisplatin and etoposide (Fig. 5A-5C and Fig. 5G-5I, respectively), but significantly promoted caspase-3 activation at stages earlier than that induced by camptothecin (Fig. 5D-5F).

In summary, although previous studies detected caspase-3 activation using different FRET sensors (Wu et al., 2006) or traditional biological methods (Namura et al., 1998), they did not report the dynamics of caspase-3 during cancer cell apoptosis. In the current study, the dynamics of caspase-3 activation in three cancer cell lines treated with chemotherapy drugs were compared for the first time using CE. The results of the present study provide a reliable reference for future studies on the molecular mechanisms of apoptosis initiation. Moreover, the results introduce an anticancer drug screening model for future clinical use.

\section{MATERIALS AND METHODS}

\section{Cell lines and reagents}

Construction of the caspase-3 FRET probe CD3, as well as a stable Hela-CD3 cell line, is as described in a previous study (Lin et al., 2006). ACC-M and HepG2 cells were obtained from China Typical Culture Collection Center (Wuhan University, Wuhan, China), and maintained in a DMEM (Gibco, USA) medium supplemented with $10 \%$ newborn calf serum (Gibco), penicillin (100 U/mL), and streptomycin $(100 \mu \mathrm{g} / \mathrm{mL})$ in culture flasks containing $5 \% \mathrm{CO}_{2}$ at $37^{\circ} \mathrm{C}$. The CD3 plasmid was transfected into the HepG2 and ACC-M cells using Lipofectamine 2000 (Invitrogen) according to the manufacturer's protocol to establish a FRET sensor expressing HepG2 and ACC-M cell lines. After $24 \mathrm{~h}$ of transfection, $800 \mu \mathrm{g} / \mathrm{mL}$ of $\mathrm{G} 418$ (Sigma) was added into the cells, and the G418 concentration was adjusted according to cell viability. Clones stably expressing HepG2-CD3 and ACC-M-CD3 were selected using G418.

Cisplatin, hydroxy-camptothecin, and etoposide injections were purchased from a pharmaceutical factory (Dezhou, China), Feiyun Pharmaceutical Company (Huangshi, China), and Hengrui Medicine Company (Jiangsu, China), respectively. Saida Bio-pharmaceutical Company (Shanghai, China) provided the rmhTNF used in the present work. Uncoated fused-silica capillary tubes (i.d. $50 \mu \mathrm{m}$, o.d. $365 \mu \mathrm{m}$ ) were obtained from Yongnian Optic Fiber Inc. (China).

\section{Cell apoptosis induced by drug treatment}

Hela-CD3, HepG2-CD3, and ACC-M-CD3 cells were placed in 24-well plates $\left(2 \times 10^{5}\right.$ cells/well) for the independent drug treatments. Adherent cells were incubated with serum-starved DMEM and desired concentrations of anticancer drugs cisplatin, camptothecin, and etoposide, respectively, for different time points. The concentration of each drug was varied accordingly as follows: cisplatin: 17, 33, 50, and $67 \mu \mathrm{mol} / \mathrm{L}$; camptothecine: 10, 20, 30, 40, and $50 \mu \mathrm{mol} / \mathrm{L}$; and etoposide: 50,100 , and $150 \mu \mathrm{mol} / \mathrm{L}$. Concentrations of $67 \mu \mathrm{mol} / \mathrm{L}$ cisplatin, $30 \mu \mathrm{mol} / \mathrm{L}$ camptothecin, and $50 \mu \mathrm{mol} / \mathrm{L}$ etoposide were selected as the most efficient concentrations for inducing cell apoptosis. Each drug was added into the reservoirs of the well plates at different time points, and a reservoir with medium alone was set as the control group.

Three cell lines were cultured in 24 -well plates $\left(2 \times 10^{5}\right.$ cells/well). 
Each line was divided into three groups and then treated with $1 \times 10^{4} \mathrm{IU} / \mathrm{mL} \mathrm{TNF}$, a designated antitumor drug, or a TNF + drug combination.

\section{Capillary electrophoresis sample preparation}

The sample preparation for CE is described in a previous study (Zhou et al., 2006b). In brief, after drug treatment, cells were washed with cold PBS thrice and then lysed with $30 \mu \mathrm{L}$ of $0.5 \%$ Trition for $1.5 \mathrm{~min}$. The lysate was collected and centrifuged at $12,000 \mathrm{rpm}$ for $10 \mathrm{~min}$, and then the supernatant was collected as a cytosolic extract for direct use in CE.

\section{Capillary electrophoresis detection system}

Drug-induced cell apoptosis analysis was performed using a homemade CE system, as described in a previous study (Zhou et al., 2006a). The homemade CE system consisted of an inverted fluorescence microscope (Olympus IX70, Japan), a cooled charge-coupled device (CCD, Micromax $5 \mathrm{MHz}$, Roper Scientific), a camera as the detector, and a high-voltage power supply (Shanghai Nuclear Research Institute, China). A $100 \mathrm{~mW}$ high-pressure mercury lamp was used as an excitation light source. The $40 \mathrm{~cm}$ long capillary was used to detect the window, and a new capillary was washed with $0.1 \mathrm{~mol} / \mathrm{L} \mathrm{HCl}, d_{d d} \mathrm{H}_{2} \mathrm{O}, 0.1 \mathrm{~mol} / \mathrm{L} \mathrm{NaOH}$, and $\mathrm{ddH}_{2} \mathrm{O}$ for $15 \mathrm{~min}$ in sequence. Phosphate buffer $\left(0.2 \mathrm{~mol} / \mathrm{L} \quad \mathrm{KH}_{2} \mathrm{PO}_{4}, 0.2 \mathrm{~mol} / \mathrm{L}\right.$ $\mathrm{Na}_{2} \mathrm{HPO}_{4} \cdot 12 \mathrm{H}_{2} \mathrm{O}$, and $\mathrm{pH}$ value of 8.0 ) was injected into one end of the capillary using a syringe for 20 to $30 \mathrm{~min}$. Both ends of the capillary were then put into two tubes filled with $\mathrm{pH} 8.0$ phosphate buffer. The first tube was fixed close to the high voltage power source and the second one was kept far away; both tubes were maintained at the same level. The terminus of the capillary near the high voltage power source was inserted into a tube filled with the sample. The sample was injected under the pressure within 10 to $15 \mathrm{~s}$. The voltage was allowed to reach $16 \mathrm{kV}$ (Zhou et al., 2006b). The capillary was then taken as a bright field to select a ROI area for imaging. The data were collected and analyzed using WinView32 and Sigma Plot 10.0 software.

\section{CD3 FRET sensor fluorescence imaging detection}

The CD3 probe was constructed by the fusion of ECFP and DsRed fluorescent proteins linked with a DEVD amino acid recognition sequence of caspase-3, as described in a previous study (Lin et al., 2006). Cell apoptosis began upon caspase- 3 activation, followed by the attenuation of FRET. Caspase-3 activity can be monitored by detecting the signals of FRET. The three-channel FRET fluorescence imaging system was constructed in our laboratory. The system was composed of an IX70 inverted fluorescence microscope (Olympus, Japan), a $100 \mathrm{~W}$ mercury light (Olympus, Japan), a charge-coupled device (CCD, Roper Scientific, USA), and a WinView32 software (Roper Scientific). The FRET filter group had three components, namely, the CFP channel (425-445HQ DM450 460-510HQ Olympus), the DsRed channel (BP510-550 DM570 BA590 Olympus), and the FRET channel (436/20X 455DCLP 620/60M Omega Optical). The DsRed channel was used for fluorescent imaging with exposure and interval time of $280 \mathrm{~ms}$ and $2 \mathrm{~s}$, respectively.

\section{ACKNOWLEDGEMENTS}

This work was supported by the National Natural Science Foundation of China (Grant Nos. 30800339 and 30800208).

\section{ABBREVIATIONS}

CE, capillary electrophoresis; CFP, cyan fluorescence protein; FP, fluorescence protein; FRET, fluorescence resonance energy transfer; i.d., inside diameter; o.d., outside diameter; TNF, tumor necrosis factor; YFP, yellow fluorescence protein

\section{REFERENCES}

Abbott, B.P., Abbott, R., Acernese, F., Adhikari, R., Ajith, P., Allen, B., Allen, G., Alshourbagy, M., Amin, R.S., Anderson, S.B., et al., and the LIGO Scientific Collaboration \& Virgo Collaboration. (2009). An upper limit on the stochastic gravitational-wave background of cosmological origin. Nature 460, 990-994.

Berezovski, M., Li, W.P., Poulter, C.D., and Krylov, S.N. (2002). Measuring the activity of farnesyltransferase by capillary electrophoresis with laser-induced fluorescence detection. Electrophoresis 23, 3398-3403.

Bergeron, S., Beauchemin, M., and Bertrand, R. (2004). Camptothecin- and etoposide-induced apoptosis in human leukemia cells is independent of cell death receptor- 3 and -4 aggregation but accelerates tumor necrosis factor-related apoptosis-inducing ligand-mediated cell death. Mol Cancer Ther 3, 1659-1669.

Burton, E.R., and Libutti, S.K. (2009). Targeting TNF-alpha for cancer therapy. J Biol 8, 85.

Chen, A.K., and Tsourka, A. (2009). Imaging RNA in living cells with molecular beacons:current perspectives and challenges. J Innovative Opt Health Sci 2, 315-324.

Chen, A.Y., and Liu, L.F. (1994). DNA topoisomerases: essential enzymes and lethal targets. Annu Rev Pharmacol Toxicol 34, 191-218.

de Jonge, M.J., Kaye, S., Verweij, J., Brock, C., Reade, S., Scurr, M., van Doorn, L., Verheij, C., Loos, W., Brindley, C., et al. (2004). Phase I and pharmacokinetic study of XR11576, an oral topoisomerase I and II inhibitor, administered on days 1-5 of a 3-weekly cycle in patients with advanced solid tumours. $\mathrm{Br} \mathrm{J}$ Cancer 91, 1459-1465.

Hara, K., Okamoto, M., Aki, T., Yagita, H., Tanaka, H., Mizukami, Y., Nakamura, H., Tomoda, A., Hamasaki, N., and Kang, D. (2005). Synergistic enhancement of TRAIL- and tumor necrosis factor alpha-induced cell death by a phenoxazine derivative. Mol Cancer Ther 4, 1121-1127.

Hsu, J.L., Chiang, P.C., and Guh, J.H. (2009). Tunicamycin induces resistance to camptothecin and etoposide in human hepatocellular carcinoma cells: role of cell-cycle arrest and GRP78. Naunyn Schmiedebergs Arch Pharmacol 380, 373-382.

Johnstone, R.W., Ruefli, A.A., and Lowe, S.W. (2002). Apoptosis: a link between cancer genetics and chemotherapy. Cell 108, 153-164.

Jones, J., Heim, R., Hare, E., Stack, J., and Pollok, B.A. (2000). Development and application of a GFP-FRET intracellular cas- 
pase assay for drug screening. J Biomol Screen 5, 307-318.

Joseph, J., Seervi, M., Sobhan, P.K., and Retnabai, S.T. (2011). High throughput ratio imaging to profile caspase activity: potential application in multiparameter high content apoptosis analysis and drug screening. PLoS One 6, e20114.

Lin, J., Zhang, Z., Zeng, S., Zhou, S., Liu, B.F., Liu, Q., Yang, J., and Luo, Q. (2006). TRAIL-induced apoptosis proceeding from caspase-3-dependent and -independent pathways in distinct HeLa cells. Biochem Biophys Res Commun 346, 1136-1141.

Locke, S., and Figeys, D. (2000). Techniques for the optimization of proteomic strategies based on head column stacking capillary electrophoresis. Anal Chem 72, 2684-2689.

Luo, K.Q., Yu, V.C., Pu, Y., and Chang, D.C. (2003). Measuring dynamics of caspase-8 activation in a single living HeLa cell during TNFalpha-induced apoptosis. Biochem Biophys Res Commun 304, 217-222.

Mahajan, N.P., Harrison-Shostak, D.C., Michaux, J., and Herman, B. (1999). Novel mutant green fluorescent protein protease substrates reveal the activation of specific caspases during apoptosis. Chem Biol 6, 401-409.

Namura, S., Zhu, J., Fink, K., Endres, M., Srinivasan, A., Tomaselli, K.J., Yuan, J., and Moskowitz, M.A. (1998). Activation and cleavage of caspase- 3 in apoptosis induced by experimental cerebral ischemia. J Neurosci 18, 3659-3668.

Neefjes, J., and Dantuma, N.P. (2004). Fluorescent probes for proteolysis: tools for drug discovery. Nat Rev Drug Discov 3, 58-69.

Postiglione, I., Chiaviello, A., and Palumbo, G. (2011). Enhancing Photodynamyc Therapy Efficacy by Combination Therapy: Dated, Current and Oncoming Strategies. Cancers 3, 2597-2629.

Reinhold, W.C., Kouros-Mehr, H., Kohn, K.W., Maunakea, A.K., Lababidi, S., Roschke, A., Stover, K., Alexander, J., Pantazis, P., Miller, L., et al. (2003). Apoptotic susceptibility of cancer cells selected for camptothecin resistance: gene expression profiling, functional analysis, and molecular interaction mapping. Cancer Res 63, 1000-1011.

Szlosarek, P.W., and Balkwill, F.R. (2003). Tumour necrosis factor alpha: a potential target for the therapy of solid tumours. Lancet Oncol 4, 565-573.

Tsuruo, T., Naito, M., Tomida, A., Fujita, N., Mashima, T., Sakamoto, H., and Haga, N. (2003). Molecular targeting therapy of cancer: drug resistance, apoptosis and survival signal. Cancer Sci 94,
15-21.

Tyas, L., Brophy, V.A., Pope, A., Rivett, A.J., and Tavaré, J.M. (2000). Rapid caspase-3 activation during apoptosis revealed using fluorescence-resonance energy transfer. EMBO Rep 1, 266-270.

Wu, Y.X., Xing, D., Luo, S.M., Tang, Y.H., and Chen, Q. (2006). Detection of caspase- 3 activation in single cells by fluorescence resonance energy transfer during photodynamic therapy induced apoptosis. Cancer Lett 235, 239-247.

Yang, J., Zhang, Z., Lin, J., Lu, J., Liu, B.F., Zeng, S., and Luo, Q. (2007). Detection of MMP activity in living cells by a genetically encoded surface-displayed FRET sensor. Biochim Biophys Acta 1773, 400-407.

Yao, X., Panichpisal, K., Kurtzman, N., and Nugent, K. (2007). Cisplatin nephrotoxicity: a review. Am J Med Sci 334, 115-124.

Zarrine-Afsar, A., and Krylov, S.N. (2003). Use of capillary electrophoresis and endogenous fluorescent substrate to monitor intracellular activation of protein kinase A. Anal Chem 75, 3720-3724.

Zhang, Z., Lin, J., Chu, J., Ma, Y., Zeng, S., and Luo, Q. (2008a). Activation of caspase- 3 noninvolved in the bystander effect of the herpes simplex virus thymidine kinase gene/ganciclovir (HSV-tk/GCV) system. J Biomed Opt 13, 031209.

Zhang, Z., Yang, J., Lu, J., Lin, J., Zeng, S., and Luo, Q. (2008b). Fluorescence imaging to assess the matrix metalloproteinase activity and its inhibitor in vivo. J Biomed Opt 13, 011006.

Zhou, S., Lin, J., Du, W., Zhang, Z., Luo, Q., Liu, B.F., and Dai, Y. (2006a). Characterization of proteinase activation dynamics by capillary electrophoresis conjugating with fluorescent protein-based probe. J Chromatogr B Analyt Technol Biomed Life Sci 844, 158-162.

Zhou, S.X., Lin, J.Q., Du, W., Zhang, Z.H., Luo, Q.M., Liu, B.F., and Dai, Y.Q. (2006b). Monitoring of proteinase activation in cell apoptosis by capillary electrophoresis with bioengineered fluorescent probe. Anal Chim Acta 569, 176-181.

Zhu, L., Liu, T.C.-Y., Wu, M., Yuan, J.-Q., and Chen, T.-S. (2009). Extraocular Cellular Phototransduction. J Innovative Opt Health Sci 2, 93-100.

Zuryn, A., Grzanka, A., Stepien, A., Grzanka, D., Debski, R., and Smolinski, D. (2007). Expression of cyclin A in human leukemia cell line HL-60 following treatment with doxorubicin and etoposide: the potential involvement of cyclin A in apoptosis. Oncol Rep 17, 1013-1019. 